\title{
Expiratory muscle training and sensation of respiratory effort during exercise in normal subjects
}

\author{
Shunsuke Suzuki, Masamichi Sato, Takao Okubo
}

\begin{abstract}
Background - The sensation of respiratory effort may increase as expiratory muscles become fatigued during expiratory loading. A study was performed to determine whether expiratory muscle training (EMT) affects the sensation of respiratory effort during exercise in healthy subjects. Methods - Six subjects performed EMT for 15 minutes twice daily for four weeks using a pressure threshold device; another six subjects served as a control group. The expiratory threshold was set at $30 \%$ of the individual's maximum expiratory mouth pressure (PEmax). The sensation of respiratory effort was evaluated during a progressive exercise test using the Borg scale.
\end{abstract}

Results - After EMT PEmax increased by $25 \%$ in the training group. The Borg score increased as exercise grade increased before and after EMT, but scores for each grade were lower after EMT. Minute ventilation during exercise decreased after EMT, as did the breathing frequency during exercise, while the expiratory time increased. Although there was no difference in the relationship between Borg score and minute ventilation before or after EMT, the curve shifted to a lower Borg score after EMT. There were no changes in PEmax, Borg score, minute ventilation, or breathing pattern after the four week study period in the control group.

Conclusion - These findings suggest that EMT increases expiratory muscle strength and reduces the sensation of respiratory effort during exercise, presumably by reducing minute ventilation.

(Thorax 1995;50:366-370)

The First Department of Internal Medicine, Yokohama City

University School of

Medicine,

3-9 Fukuura,

Kanazawa-ku,

Yokohama,

Kanagawa 236, Japan

S Suzuki

M Sato

T Okubo

Reprint requests to: Dr S Suzuki.

Received 18 April 1994

Returned to authors

5 September 1994

Revised version received

7 November 1994

Accepted for publication

5 January 1995
Keywords: expiratory muscle training, respiratory effort sensation, exercise, minute ventilation. dyspnoea is determined by the magnitude of the central motor command signal ${ }^{12}$ which increases whenever the peripheral muscles become weak or fatigued. ${ }^{1-3}$ Respiratory muscle fatigue increases the sense of effort during loaded breathing in healthy subjects. ${ }^{14}$ In patients with chronic obstructive pulmonary disease (COPD) the expiratory muscles become weakened, but to a lesser extent than the inspiratory muscles. ${ }^{5}$ Experimentally, resistive loaded breathing on expiration has caused ex-
It has been suggested that the sensation of piratory fatigue in healthy subjects. ${ }^{6}$ We recently found that the sensation of respiratory effort increased as expiratory muscles became fatigued during expiratory loading. ${ }^{7}$

Leith and Bradley reported that respiratory muscle training increases respiratory muscle strength in normal subjects, ${ }^{8}$ and inspiratory muscle training improves inspiratory muscle strength as well as exercise endurance in patients with COPD. ${ }^{9}{ }^{10}$ However, several questions concerning the effect of respiratory muscle training in patients with COPD are raised. ${ }^{112}$ Recently, we found no improvement in the sensation of respiratory effort during exercise after inspiratory muscle training in normal subjects. ${ }^{13}$ It is not clear whether improved respiratory muscle strength influences the sensation of respiratory effort, although respiratory muscle fatigue increases the sensation of respiratory effort. ${ }^{147}$ During exercise expiratory muscles, especially the abdominal muscles, contribute substantially to increased ventilation; the work of inspiratory muscles also increases during exercise. ${ }^{1415}$ An increased sensation of respiratory effort during exercise may therefore be related to the participation of expiratory muscles.

We hypothesised that, during increased ventilation such as exercise, an increase in expiratory muscle strength would reduce the magnitude of the central command needed for a given amount of work from the expiratory muscles, thereby reducing the sensation of respiratory effort. To examine this hypothesis we measured the sensation of respiratory effort in healthy subjects during a progressive exercise test before and after a four week period of expiratory muscle training using a pressure threshold device.

\section{Methods}

Twelve healthy volunteers ( 10 men, mean (SD) age $30.0(5.5)$ years (range 24 to 44 )) took part in the study. All were non-smokers with no history of chronic respiratory or circulatory disease. Subjects were randomly assigned to control $(n=6 ; 5$ men) or training groups $(n=$ 6; 5 men). Informed consent was obtained from each subject. The study was approved by the committee on human investigation of our institution.

On test days subjects were prohibited from drinking coffee or other caffeine-containing beverages. Spirometric measurements were performed using a dry seal spirometer (OST-80, Chest Co, Tokyo, Japan), and vital capacity 
(VC) and forced expiratory volume in one second $\left(\mathrm{FEV}_{1}\right)$ were measured. The maximal voluntary ventilation (MVV) was determined from maximal voluntary ventilation over a 15 second period. Lung volumes were measured using a body plethysmograph (Autobox 2800, Gould, Dayton, Ohio, USA).

\section{MAXIMUM RESPIRATORY PRESSURE}

Maximum static inspiratory and expiratory mouth pressures (PImax and Pemax) were measured according to the method of Black and Hyatt. ${ }^{16}$ Briefly, mouth pressure was measured using a differential pressure transducer (Validyne MP-45 $\pm 250 \mathrm{~mm} \mathrm{Hg}$, Northridge, California, USA). PImax was determined from the maximal inspiration against a closed valve at functional residual capacity (FRC) and residual volume (RV). PEmax was determined from the maximal expiration against a closed valve at FRC and total lung capacity (TLC) while subjects pressed their cheeks with their hands. They practised these pressure measurements two days before baseline measurements were obtained (control day) to reduce any learning effect. On test days PImax and Pemax were measured at least three times until a reproducible value was obtained and maximum values were reported.

\section{RESPIRATORY EFFORT DURING EXERCISE}

Respiratory effort during exercise was evaluated by a modified Borg scale. ${ }^{17}$ This scale consists of a series of integers from 0 to 10 . The subject was told to estimate the effort required to produce each breath, but not shortness of breath or the effort of exercise. During exercise the Borg scale was kept in front of the subject who was asked to rate effort at one minute intervals.

\section{EXERCISE TEST}

A progressive exercise test was performed on a treadmill (Quinton Q50, Seattle, Washington, USA). Each subject breathed air through a twoway non-rebreathing valve (No. 2700, Hans Rudolph, Kansas City, Missouri, USA) so that concentrations of expired gas and ventilation could be measured. Expired gas was sampled and analysed breath by breath. Tidal volume (VT), breathing frequency, minute ventilation $(\dot{V E})$, oxygen uptake $\left(\dot{\mathrm{VO}}_{2}\right)$, output of carbon dioxide $\left(\mathrm{VCO}_{2}\right)$, and dead space/tidal volume ratio (VD/VT) were determined using the 2900 Energy Measurement System (Sensormedics, Anaheim, California, USA). Expiratory time (Te) was calculated from the flow signal. The $\mathrm{VD} / \mathrm{VT}$ was determined using the Bohr equation. Arterial oxygen saturation was measured with a pulse oximeter (Biox 3740, Ohmeda, Boulder, Colorado, USA). An electrocardiogram was monitored throughout the experiment.

A modified Balke exercise protocol was used. ${ }^{18}$ The workload was increased by approximately 1 met (metabolic equivalent of resting $\mathrm{O}_{2}$ uptake) at one minute intervals. At stage 1 the treadmill was kept at a constant speed of $2.0 \mathrm{mph}$ and the grade at $0 \%$. Beginning at stage 2 , the grade was increased from $0 \%$ by $2 \cdot 5 \%$ at one minute intervals that is, one stage per minute - up to stage 10 , and the speed was kept at a constant $3.3 \mathrm{mph}$. The exercise test was continued until the subject reached a state of exhaustion or stage 10 with a slope of $20 \%$.

\section{EXPIRATORY MUSCLE TRAINING (EMT)}

Subjects in the training group trained with a threshold pressure breathing device (Threshold Inspiratory Muscle Trainer, Healthscan Products, Cedar Grove, New Jersey, USA) that uses a spring loaded poppet valve. We modified its spring to produce pressure loads ranging from 15 to $125 \mathrm{~cm} \mathrm{H}_{2} \mathrm{O}$. This device is designed primarily for inspiratory muscle training. We modified the conventional method of using the device by asking the subjects to expire through the opposite side of the device. The threshold pressure used was equal to $30 \%$ of the individual's PEmax at TLC before EMT and was kept constant throughout the four week training period. Subjects were instructed to expire air twice daily through the threshold device at their own breathing frequency and tidal volume for 15 minutes and to record their execution of EMT.

\section{EXPERIMENTAL PROTOCOL}

No other form of exercise training was started during the four week study, and all subjects maintained their usual habits during the study period. On day 1 spirometric parameters, lung volumes, PImax, and Pemax were determined to obtain baseline values. The sensation of respiratory effort was measured during the treadmill exercise test. After two weeks of EMT, PImax and Pemax were measured, and spirometric measurements and the exercise test were repeated. After four weeks of EMT spirometric measurements were again made, and lung volumes, maximum respiratory pressures, and the sensation of respiratory effort were determined during exercise.

\section{DATA ANALYSIS}

All values were reported as the mean (SD). Differences in respiratory muscle strength between groups were analysed by the unpaired $t$ test. $^{19}$ Spirometric values and respiratory muscle strength before and after EMT were analysed by the paired $t$ test. The Borg score was analysed using Friedman's test. Ventilation parameters were analysed using two-way analysis of variance with repeated measures (ANOVA), followed by post hoc comparison using the Tukey's test. A p value of less than 0.05 was considered statistically significant.

\section{Results}

All subjects in the training group completed the study. Vital capacity, $\mathrm{FEV}_{1}$, lung volumes, and MVV did not change over the four week 
Control group

Training group

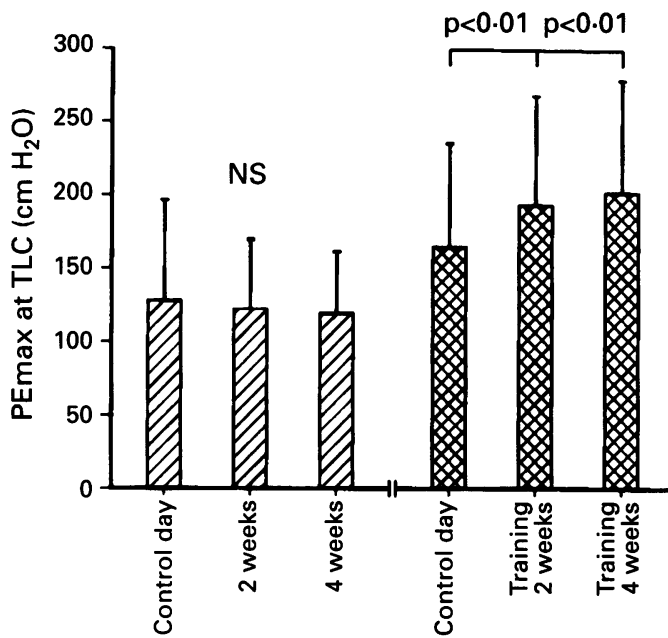

Figure 1 Mean (SD) expiratory muscle strength before and after expiratory muscle strength training. In the training group an increase in maximum expiratory muscle strength (PEmax at TLC) was observed at two and four weeks (both $p<0 \cdot 01$ ). There was no change in the control group.

study period in either the control or the training group. PEmax at TLC and FRC and PImax at FRC and RV were within normal limits in both groups. There were no differences in PEmax and PImax before EMT between the two groups. In the training group, Pemax at TLC increased significantly compared with
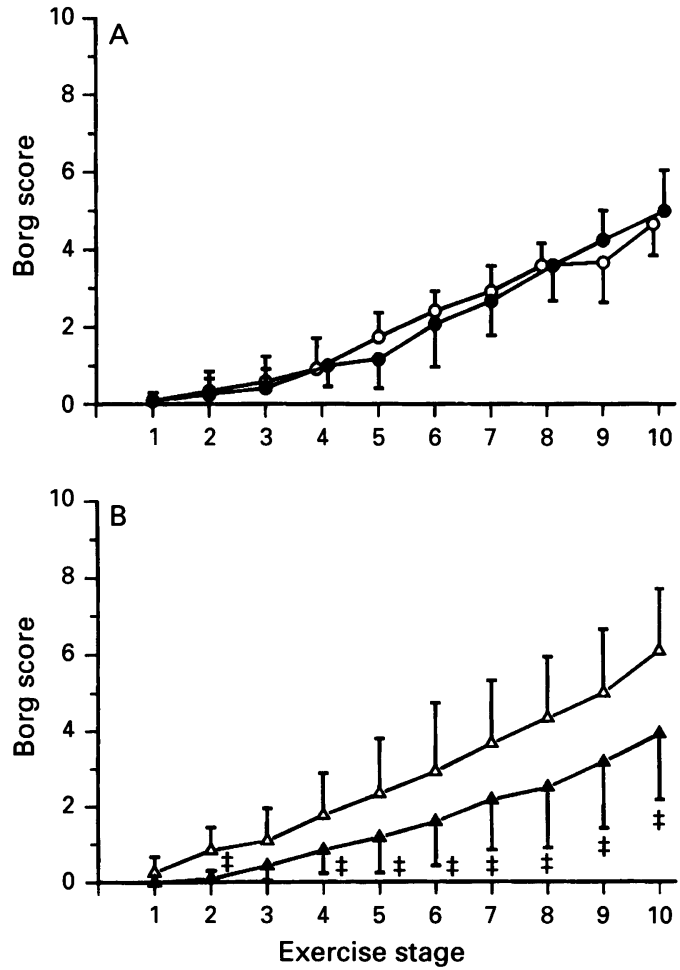

Figure 2 Relationship between Borg score and exercise stage in $(A)$ control $(n=6)$ and $(B)$ training $(n=6)$ groups on the control day $(O, \triangle)$ and after the four week study period $(\mathbf{O}, \mathbf{\Delta})$. The Borg score in the training group decreased at four weeks but there was no change in Borg score in the control group. $\ddagger p<0.01$, compared with control day (Friedman's test). Values are mean (SD). control day values at two weeks (average $20.0 \%$ ) and four weeks (from 165(71) to $202(77) \mathrm{cm} \mathrm{H}_{2} \mathrm{O}$; average increase $25.4 \%$ ) $(\mathrm{p}<0.01$ ) (fig 1). PEmax at FRC increased by $20 \cdot 2 \%$ after two weeks of EMT $(p<0.01)$ and by $18 \cdot 2 \%$ after four weeks (from $143(65)$ on the control day to $\left.168(72) \mathrm{cm} \mathrm{H}_{2} \mathrm{O}, \mathrm{p}<0.05\right)$. Individual differences in PEmax did not change after EMT. PImax did not change at RV or FRC. There were no significant changes in PEmax or PImax at any lung volume in the control group.

All subjects completed the exercise test to stage 10 on both the control day and after four weeks, and consequently the exercise test was submaximal exercise. Before EMT the Borg score increased linearly with time in both groups $(r=0.96(0.05)$ and $0.97(0.04)$ for the control and training groups, respectively) (fig $2)$. On the control day there was no significant difference between the two groups in the Borg score at any stage of exercise. After EMT the Borg score was significantly lower than the control day at each exercise stage (fig 2, $\mathrm{p}<0 \cdot 01)$. In the control group the Borg score did not change significantly at any exercise stage during the four week study period. The slope of the Borg score with time (or exercise stage) decreased from $0.63(0.17) \mathrm{min}^{-1}$ to $0.43(0.22) \mathrm{min}^{-1}$ at four weeks $(\mathrm{p}<0.05)$ in the training group, but did not change in the control group.

Minute ventilation ( $\dot{V} E$ ) increased with exercise stage in both groups (fig 3 ). In the

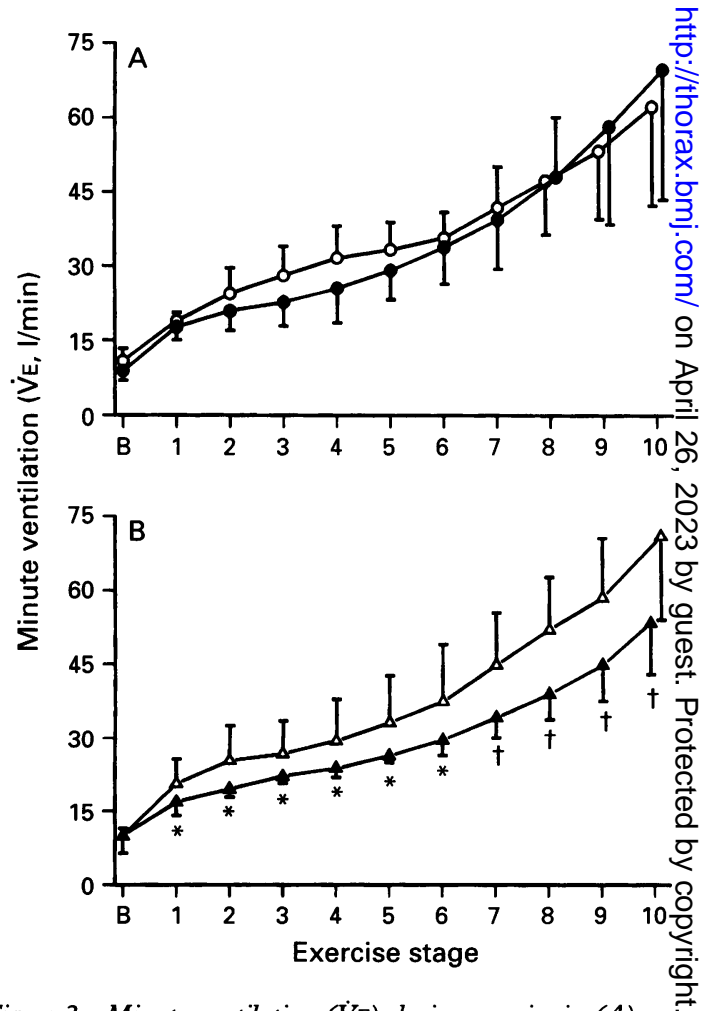

Figure 3 Minute ventilation ( $\dot{V} E)$ during exercise in $(A)$ the control group $(n=6)$ and $(B)$ the training group $(n=$ 6) on the control day $(O, \triangle)$ and after the four week study period $(\mathbf{O}, \mathbf{\Delta})$. After training the $\dot{V}_{E}$ decreased ( $p<0.05$, ANOVA) but there was no difference between control day and four week values in the control group. ${ }^{*} p<0.05,+p<0.02$, compared with control day (Tukey's test). Values are mean (SD). 

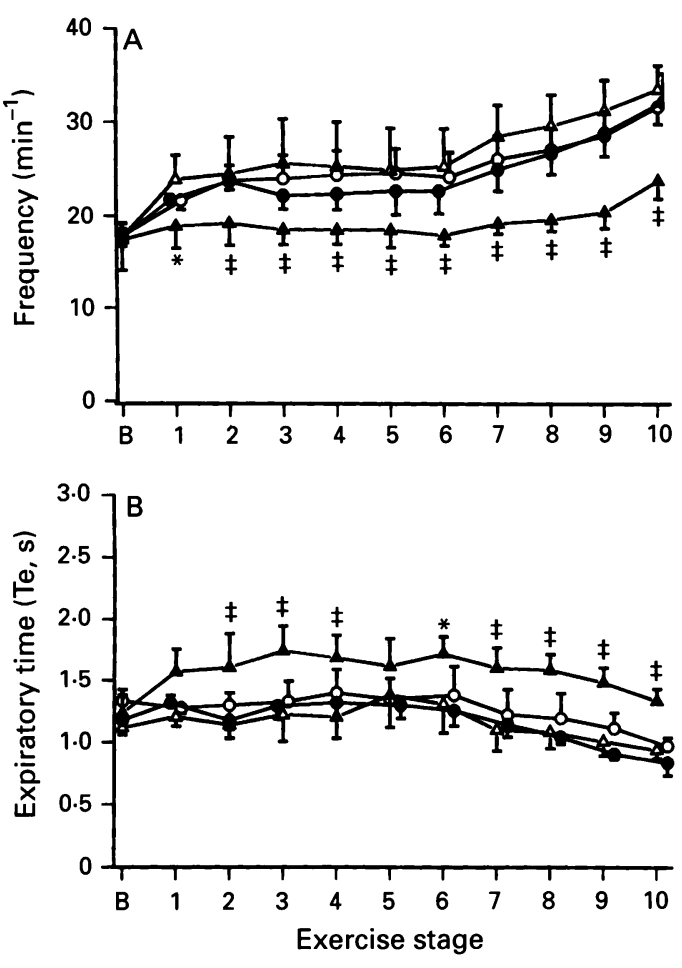

Figure 4 (A) Breathing frequency and (B) expiratory time (Te) during exercise of control and training groups on the control day $(O, \triangle)$ and after the four week study period $(\mathbf{O}, \mathbf{\Delta})$. Expiratory muscle training reduced breathing frequency $(p<0 \cdot 05, A N O V A)$ and increased Te $(p<0.05, A N O V A)$, although the Te decreased with exercise stage in the control group. ${ }^{*} p<0.05, \ddagger p<0.01$ compared with control day (Tukey's test). Values are mean (SE).

training group $\dot{V} E$ was significantly lower after EMT than on the control day $(p<0.05$, ANOVA). VE did not change in the control group. The VT increased with exercise stage in both groups and did not change after EMT. Breathing frequency increased with exercise in both groups (fig 4). In the training group breathing frequency was lower after EMT compared with the pre-EMT value $(\mathrm{p}<0.05$, ANOVA). The expiratory time (Te) decreased with exercise stage in both groups (fig 4). In the training group the Te increased after EMT $(p<0.05$, ANOVA). In the control group there was no significant difference in Te between the control day and the four week values. The $\mathrm{VD} /$ VT decreased with increasing exercise stage. There was no difference in VD/VT between the control day and the four week values in either group. $\dot{\mathrm{VO}}_{2}$ and $\dot{\mathrm{V}} \mathrm{CO}_{2}$ increased progressively with exercise in both groups. $\mathrm{VO}_{2}$ and $\dot{\mathrm{V}} \mathrm{CO}_{2}$ tended to decrease after EMT, but the difference was not significant. The ventilatory equivalent for oxygen $\left(\dot{\mathrm{V}} \mathrm{E} / \mathrm{Vo}_{2}\right)$ decreased towards the midpoint of the exercise protocol and then increased in both groups. EMT reduced VE/ $\mathrm{VO}_{2}$ in the second half of the exercise protocol $(p<0.05)$. There was no change in arterial oxygen saturation during exercise on both the control day and after four weeks in either group.

The Borg score and VE were closely correlated in all subjects on each test day $(0.89<r<0.99)$. The relationship between the Borg score and the $\dot{V}$ was similar on the control day and after four weeks in both groups.
In the training group EMT shifted the position of the curve toward a lower Borg score without any change in relationship of Borg score to $\dot{\mathrm{VO}}_{2}$, but in the control group the position of the curve did not change.

\section{Discussion}

We have shown that a four week period of EMT using a pressure threshold device increased the strength of the expiratory muscles and reduced the sensation of respiratory effort during exercise in normal subjects. The relation of the sensation of respiratory effort to $\dot{V} E$ was not changed by EMT, although the sensation of respiratory effort and VE decreased after EMT at each exercise stage, suggesting that EMT diminishes the sensation of respiratory effort during exercise by reducing $\dot{V} E$.

\section{EXPIRATORY MUSCLE TRAINING (EMT)}

Leith and Bradley demonstrated that ventilatory muscle training increased ventilatory muscle strength. ${ }^{8}$ Inspiratory muscle training has been attempted in patients with COPD, 910 but no previous study has investigated the effect of EMT using expiratory loading. In the present study EMT was performed using pressure threshold loading at $30 \%$ of the subject's PEmax at TLC. All subjects in the training group showed an increase in PEmax after EMT. We expected that an increase of this magnitude in expiratory muscle strength would allow us to examine the effects of strengthening of respiratory muscles on the sensation of respiratory effort.

\section{SENSATION OF RESPIRATORY EFFORT DURING EXERCISE}

While patients with COPD experience breathlessness more intensely during inspiration, ${ }^{20}$ the sensation generated during exercise in normal subjects is more intense during expiration. ${ }^{21}$ During exercise expiratory muscles, especially the abdominal muscles, contribute substantially to increased ventilation, ${ }^{14}{ }^{15}$ although the work of inspiratory muscles also increases. The sensation generated during exercise is closely related to factors that contribute to respiratory muscle effort such as increased ventilation. Minute ventilation represents the overall output of the respiratory system..$^{22}$ During exercise consistently linear relationships have been observed between $\dot{V}_{E}$ and the intensity of breathing-related sensation in normal subjects. $^{2223}$ In the present study the relationship between Borg score and VE did not change over four weeks in either study group, suggesting that $\dot{V} E$ may be one of the most useful parameters for evaluating the sensation of respiratory effort during treadmill exercise.

EFFECT OF EMT ON THE SENSATION OF RESPIRATORY EFFORT

We expected that an enhancement of expiratory muscle strength would reduce the fraction of expiratory muscle force needed for a given 
amount of work, which would reduce the magnitude of the central motor command to expiratory muscles, thereby reducing the sensation of respiratory effort. If a decrease in the fraction of expiratory muscle force during expiration directly affected the sensation of respiratory effort, the relationship of Borg score to $\dot{V} E$ would have been changed by EMT. In the present study the relationship of Borg score to VE was unchanged by EMT, although expiratory muscle strength was increased by EMT. We therefore concluded that an increase in expiratory strength may not directly affect the sensation of respiratory effort during exercise.

Abdominal muscles, which are major expiratory muscles, were trained by EMT in the subjects studied. In addition to contributing to expiration, abdominal muscles work to stabilise the body position during exercise. It is possible, therefore, that EMT may reduce the $\dot{\mathrm{VO}}_{2}$ of the abdominal muscles for both these functions during exercise. Also, physical training is known to reduce both $\dot{\mathrm{VE}}$ and $\dot{\mathrm{Vo}}_{2}$ of whole body. ${ }^{24} 25$ reduction in $\dot{V}$ may reduce the sensation of respiratory effort at each exercise stage.

In the present study EMT altered breathing patterns, such as breathing frequency, $\mathrm{Te}$ and VT. The Te decreased with increasing exercise stage in the control group but increased after EMT in the training group. Burdon and coworkers showed that, during exercise, the breathing frequency increased; the Te showed a greater decrease than the inspiratory time. ${ }^{26}$ In our study EMT may have increased Te during breathing through expiratory loading to reduce the difficulty of breathing because subjects were asked to perform EMT using their own breathing patterns. Belman and coworkers showed that a change in breathing pattern improved ventilatory muscular performance. ${ }^{27}$ Thus, our subjects may have learned a breathing strategy that lengthened Te in the presence of increased work of breathing. A decrease in breathing frequency associated with lengthened Te may reduce $\mathrm{VE}$. However, EMT altered the relationship of Borg score to $\mathrm{Te}$ and the breathing frequency. These findings suggest that the EMT-induced change in breathing pattern may not directly affect the sensation of respiratory effort during exercise.

Although EMT produced no change in $\mathrm{VD} /$ $\mathrm{VT}$, it reduced the equivalent for oxygen $\left(\dot{V}_{\mathrm{E}} /\right.$ $\dot{V O}_{2}$ ), suggesting that it enhanced the effectiveness of ventilation during exercise. Increases in Te and Pemax may permit the expiratory muscles to play a major part in expiration during exercise, lengthening the diaphragm and improving the length-tension characteristics for generation of pressure required to produce a subsequent inspiration. ${ }^{141528}$ Thus, EMT may improve diaphragmatic function, reducing the sensation of respiratory effort.

In conclusion, expiratory muscle training reduces the sensation of respiratory effort during exercise in normal subjects, although the exact mechanism of this effect is unclear. Before this expiratory muscle training programme can be applied to patients with respiratory disease, the effect of EMT on breathlessness should be investigated in a modified programme for patients.

1 Gandevia SC, Killian KJ, Campbell EJM. The effect of respiratory muscle fatigue on respiratory sensations. Clin Sci 1981;60:463-6.

2 Killian KJ, Campbell EJM. Dyspnea. In: Roussos C, Macklem PT, eds. The Thorax. New York: Marcel Dekker, 1985:787-882.

3 Campbell EJM, Gandevia SC, Killian KJ, Manhutte CK. Changes in the perception of inspiratory resistive loads during partial curarization. $\mathcal{f}$ Physiol Lond 1980;309:93100.

4 Supinski GS, Clary SJ, Bark H, Kelsen SG. Effect of inspiratory muscle fatigue on perception of effort during loaded breathing. F Appl Physiol 1987;62:300-7.

5 Rochester DF, Braun NM, Arora NS. Respiratory muscle strength in chronic obstructive pulmonary disease. $\mathrm{Am}$ Rev Respir Dis 1979;119:151-4.

6 Suzuki S, Suzuki J, Okubo T. Expiratory muscle fatigue in normal subjects. f Appl Physiol 1991;70:2632-9.

7 Suzuki S, Suzuki J, Ishii T, Akahori T, Okubo T. Relationship of respiratory effort sensation to expiratory muscle fatigue during expiratory threshold loading. $\mathrm{Am}$ muscle fatigue during expiratory

8 Leith DE, Bradley $M$. Ventilatory muscle strength and endurance training. F Appl Physiol 1976;41:508-16.

9 Chen H, Dukes R, Martin BJ. Inspiratory muscle training in patients with chronic obstructive pulmonary disease. Am Rev Respir Dis 1985;131:251-5.

10 Larson JL, Kim MJ, Sharp JT, Larson DA. Inspiratory muscle training with a pressure threshold breathing device in patients with chronic obstructive pulmonary disease. Am Rev Respir Dis 1988;138:689-96.

11 Smith K, Cook D, Guyatt GH, Madhavan J, Oxman AD. Respiratory muscle training in chronic airflow limitation: a meta-analysis. Am Rev Respir Dis 1992;145:533-9.

12 Levine S, Weiser P, Gillen J. Evaluation of a ventilatory muscle endurance training program in the rehabilitation of patients with chronic obstructive pulmonary disease. Am Rev Respir Dis 1986;133:400-6.

13 Suzuki S, Yoshiike Y, Suzuki M, Akahori T, Hasegawa A, Okubo $\mathrm{T}$. Inspiratory muscle training and respiratory sensation during treadmill exercise. Chest 1993;104:197202.

14 Grimby G, Goldman M, Mead J. Respiratory muscle action inferred from rib cage and abdominal V-P partitioning. $f$ Appl Physiol 1976;41:739-51.

15 Goldman MD, Grassino A, Mead J, Sears A. Mechanics of the human diaphragm during voluntary contraction: dynamics. $\mathcal{F}$ Appl Physiol 1978;44:840-8.

16 Black LF, Hyatt RE. Maximal respiratory pressures: norma values and relationship to age and sex. Am Rev Respir Dis 1969;99:696-702.

17 Borg GAV. Psychophysical bases of perceived exertion. Med Sci Sports Exerc 1982;14:377-81.

18 Nagle FJ, Balke B, Naughton JP. Gradational step tests for assessing work capacity. 7 Appl Physiol 1965;20:745-8.

19 Wampold BE, Drew CJ. Theory and application of statistics. New York: McGraw-Hill, 1990.

20 Stark RD, Lewis JA. Asthma - expiratory dyspnoea? BMF $1981 ; 2: 1121-2$.

21 Adams L, Guz A. Dyspnea on exertion. In: Whipp BJ, Wasserman $\mathrm{K}$, eds. Exercise: pulmonary physiology and pathophysiology. New York: Marcel Dekker, 1991:449-94.

22 Adams L, Chronos N, Lane R, Guz A. The measurement of breathlessness induced in normal subjects: validity of two scaling techniques. Clin Sci 1985;69:7-16.

23 Wilson RC, Jones PW. A comparison of the visual analogue scale and modified Borg scale for the measurement of dyspnoea during exercise. Clin Sci 1989;76:277-82.

24 Cotes JE, Reed JW, Elliot C. Breathing and exercise requirements of the work place. In: Whipp BJ, Wasserman $\mathrm{K}$, eds. Exercise: pulmonary physiology and pathophysiology. New York: Marcel Dekker, 1991:495-548.

25 Casaburi R, Storer TW, Wasserman K. Mediation of reduced ventilatory response to exercise after endurance training. F Appl Physiol 1987;63:1533-8.

26 Burdon JGW, Killian KJ, Jones NL. The pattern of breathing during exercise in patients with interstitial lung disease. Thorax 1983;38:778-84.

27 Belman MJ, Thomas SG, Lewis MI. Resistive breathing training in patients with chronic obstructive pulmonary disease. Chest 1986;90:662-9.

28 Jones NL, Killian KJ, Stubbing D. The thorax in exercise. In: Roussos C, Macklem PT, eds. The Thorax. New York: Marcel Dekker, 1985:627-62.

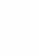

